\title{
Cardiovascular Event Rates in Statin-Treated Korean Patients with Cardiovascular Disease: Estimates from a Real-World Population Using Electronic Medical Record Data
}

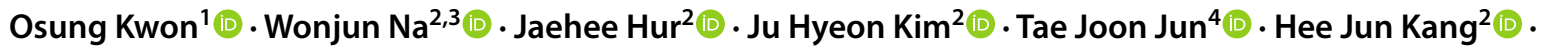 \\ Hojoon Lee ${ }^{5}$ (i) $\cdot$ Young-Hak Kim ${ }^{2}$ (i)
}

Accepted: 3 September 2021 / Published online: 8 October 2021

(c) The Author(s) 2021, corrected publication October/2021

\begin{abstract}
Purpose To estimate the risk of recurrent cardiovascular events in a real-world population of very high-risk Korean patients with prior myocardial infarction (MI), ischemic stroke (IS), or symptomatic peripheral artery disease (SPAD), similar to the Further cardiovascular OUtcomes Research with proprotein convertase subtilisin-kexin type 9 Inhibition in subjects with Elevated Risk (FOURIER) trial population.

Methods This retrospective study used the Asan Medical Center Heart Registry database built on electronic medical records (EMR) from 2000 to 2016. Patients with a history of clinically evident atherosclerotic cardiovascular disease (ASCVD) with multiple risk factors were followed up for 3 years. The primary endpoint was a composite of MI, stroke, hospitalization for unstable angina, coronary revascularization, and all-cause mortality.

Results Among 15,820 patients, the 3-year cumulative incidence of the composite primary endpoint was $15.3 \%$ and the 3-year incidence rate was 5.7 (95\% CI 5.5-5.9) per 100 person-years. At individual endpoints, the rates of deaths, MI, and IS were $0.4(0.3-0.4), 0.9(0.8-0.9)$, and $0.8(0.7-0.9)$, respectively. The risk of the primary endpoint did not differ significantly between recipients of different intensities of statin therapy. Low-density lipoprotein cholesterol (LDL-C) goals were only achieved in $24.4 \%$ of patients during the first year of follow-up.

Conclusion By analyzing EMR data representing routine practice in Korea, we found that patients with very high-risk ASCVD were at substantial risk of further cardiovascular events in 3 years. Given the observed risk of recurrent events with suboptimal lipid management by statin, additional treatment to control LDL-C might be necessary to reduce the burden of further cardiovascular events for very high-risk ASCVD patients.
\end{abstract}

Keywords Cardiovascular disease $\cdot$ Secondary prevention $\cdot$ Dyslipidemia $\cdot$ Cholesterol $\cdot$ PCSK9 inhibitors $\cdot$ Electronic medical records

Young-Hak Kim

mdyhkim@amc.seoul.kr

1 Division of Cardiology, Department of Internal Medicine, Eunpyeong St. Mary's Hospital, College of Medicine, The Catholic University of Korea, Seoul, Republic of Korea

2 Division of Cardiology, Department of Internal Medicine, Asan Medical Center, University of Ulsan College of Medicine, Seoul, Republic of Korea

3 Department of Medical Science, Asan Medical Institute of Convergence Science and Technology, Asan Medical Center, University of Ulsan College of Medicine, Seoul, Republic of Korea

4 Health Innovation Big Data Center, Asan Institute for Life Sciences, Asan Medical Center, Seoul, Republic of Korea

5 Amgen Korea, Seoul, Republic of Korea

\section{Introduction}

Cardiovascular disease (CVD) is the leading cause of death globally [1]. Patients who experience a cardiovascular event are at increased risk of further events [2-5]. Dyslipidemia is known to be a major risk factor for CVD [6,7], and management of low-density lipoprotein cholesterol (LDL-C) levels is critical in patients at high risk of CVD [8, 9]. High LDL-C is not only a risk factor for primary CVD events, but also for secondary or recurrent CVD events in high-risk CVD patients [10-12]. Statins have been the mainstay of LDL-C lowering therapy, but there is still a residual risk of CVD attributable to high LDL-C. Thus, additional drugs may be needed to achieve adequate control in some patients [8]. 
The Further cardiovascular OUtcomes Research with proprotein convertase subtilisin-kexin type 9 (PCSK9) Inhibition in subjects with Elevated Risk (FOURIER) trial demonstrated the risk of secondary CVD events among high-risk patients and showed that adding evolocumab to statin therapy reduced the risk of recurrent cardiovascular events in patients with clinically evident atherosclerotic cardiovascular disease (ASCVD) [13]. The majority of data about the risk of cardiovascular events in patients with existing CVD and new lipid-lowering treatments come from clinical trials. A few retrospective studies have estimated the risk of cardiovascular events in very high-risk patients who met the inclusion criteria of the FOURIER trial in real-world settings (FOURIER-like populations) in Europe and the USA [14-16]. As such, there is a need to obtain data from routine clinical practice, in order to explore any evidence gaps between the real-world setting and clinical trials, particularly in Asian populations.

Thus, the purpose of the current study, using electronic medical records (EMR) data from a tertiary hospital, was to obtain information about the clinical characteristics and to estimate the rates of cardiovascular outcomes in very high-risk Korean patients with prior myocardial infarction (MI), ischemic stroke (IS), or symptomatic peripheral artery disease (sPAD) who met the inclusion criteria for the FOURIER trial. In addition, we tried to evaluate the real-world treatment pattern of LDL-C for the very high-risk Korean population.

\section{Methods}

\section{Study Design}

A retrospective observational cohort study was conducted using EMR data from a large tertiary hospital in Korea. The study included adult patients at high risk of cardiovascular events and receiving statin therapy, who were followed for the occurrence of subsequent cardiovascular events. The study period was from 1 January 2000 to 30 November 2016.

Data were obtained from the Asan biomedical research environment database, which is a data warehouse system [17] for the Asan Medical Center (AMC), one of the largest medical institutions in the Republic of Korea. The Asan biomedical research environment database includes deidentified information for 4 million patients and is updated every 3 days. The AMC Heart Registry (AMC-HR) was constructed using fields of structured or unstructured EMR data extracted from the Asan biomedical research environment database using structured query language queries. The AMC-HR was standardized and validated to allow EMRbased research [18]. The AMC-HR comprised 571,157 subjects who met the inclusion criteria of inpatient or outpatient encounters in the cardiology, cardiac surgery, or emergency departments for established or suspected heart disease between 1 January 2000 and 30 November 2016. Collection of data based on implied consent was approved by the AMC institutional review board. De-identification of data was performed in line with the health insurance portability and accountability act for Korea.

\section{Study Population}

The study included patients from the AMC-HR who had a history of clinically evident cardiovascular disease (MI, IS, or SPAD) and at least 1 major risk factor or at least 2 minor risk factors (similar to the criteria for the FOURIER trial; Table 1) between 1 January 2000 and 30 November 2013. The index date was defined as the date of the first diagnosis of MI, IS, or SPAD. Definitions and associated ICD-10 or claim codes for the inclusion criteria variables are provided in Online Resource 1. The study population was stratified into three subcohorts according to the index cardiovascular event (MI, IS, and SPAD cohorts) and intensity of statin, to facilitate a detailed analysis.

Patients were excluded if they were aged $<40$ or $>85$ years, had a recent MI or stroke within 4 weeks of the index date, did not have blood LDL-C levels available, were not receiving a statin, had uncontrolled hypertension, or had a history of hemorrhagic stroke at any time.

\section{Outcomes}

The primary endpoint was a composite of MI, stroke, hospitalization for unstable angina (UA), coronary revascularization (coronary artery bypass grafting [CABG] or percutaneous coronary intervention [PCI]), and all-cause mortality at 3-year follow-up. This mirrored the primary endpoint in the FOURIER trial except that cardiovascular-related death was replaced with all-cause mortality for the current study. The secondary endpoint was a composite of MI, stroke, and all-cause mortality.

The follow-up period continued through to 30 November 2016, to ensure that all patients had the opportunity for a 3 -year follow-up evaluation. Mortality was captured through documentation of mortality in EMR based upon national health insurance information. Cardiovascular events, including MI, UA, and stroke, were captured by identifying hospitalizations with a relevant primary or secondary ICD-10 diagnosis and adjudicating the event in conjunction with related laboratory results and reports on imaging studies by cardiologists and neurologists. Repeat revascularization was identified by the presence of cost codes for CABG or PCI, with final confirmation by a cardiologist based on the notes for the procedure. Definitions and associated ICD-10 
Table 1 Key inclusion criteria for the AMC-HR study and comparison with those for the FOURIER study

\begin{tabular}{ll}
\hline FOURIER inclusion criteria [13] & AMC-HR inclusion criteria \\
\hline Major risk factors (1 required) & Major risk factors (1 required) \\
- Diabetes (type 1 or type 2) & $\bullet$ Diabetes (type 1 or type 2 ) \\
- Age $\leq 65$ and $\geq 85$ years & $\bullet$ Age $\leq 65$ and $\geq 85$ years \\
- MI or non-hemorrhagic stroke within 6 months of screening & $\bullet$ MI or IS diagnosis within last 6 months \\
- Additional diagnosis of MI or non-hemorrhagic stroke & $\bullet$ History of $>1 \mathrm{MI}$ or IS event \\
- Current daily cigarette smoking & $\bullet$ Current smoking \\
- History of sPAD & $\bullet$ History of sPAD \\
Minor risk factors $(2$ required) & Minor risk factors $(2$ required) \\
- History of non-MI-related coronary revascularization & $\bullet$ History of non-MI-related coronary revas- \\
- Residual coronary artery disease & cularization \\
- HDL-C $<40 \mathrm{mg} / \mathrm{dL}$ for men or $<50 \mathrm{mg} / \mathrm{dL}$ for women & $\bullet$ HDL-C $<40 \mathrm{mg} / \mathrm{dL}$ for men or $<50 \mathrm{mg} / \mathrm{dL}$ \\
- High-sensitivity CRP $>2.0 \mathrm{mg} / \mathrm{L}$ & for women \\
- Metabolic syndrome, defined as the presence of at least 3 of the following 5 conditions: & $\bullet$ High-sensitivity CRP $>2.0 \mathrm{mg} / \mathrm{L}$ \\
- Elevated waist circumference & $\bullet$ Metabolic syndrome, defined as the \\
- Triglycerides $\geq 150 \mathrm{mg} / \mathrm{dL}$ & presence of at least 2 of the following 4 \\
- Low HDL-C $($ as defined above) & conditions: \\
- Fasting glucose $\geq 100 \mathrm{mg} / \mathrm{dL}$ & - BMI $\geq 30^{\mathrm{a}}$ \\
- Hypertension & - Triglycerides $\geq 150 \mathrm{mg} / \mathrm{dL}$ \\
& - Low HDL-C (as defined above) \\
\hline
\end{tabular}

$A M C$-HR Asan Medical Center Heart Registry, BMI body mass index, $C A D$ coronary artery disease, $C P R D$ Clinical Practice Research Datalink, CRP C-reactive protein, FOURIER Further cardiovascular OUtcomes Research with PCSK9 Inhibition in subjects with Elevated Risk, HDL-C high-density lipoprotein cholesterol, $I S$ ischemic stroke, $M I$ myocardial infarction, SI International System of Units, $s P A D$ symptomatic peripheral artery disease

${ }^{a}$ Modified criteria for central obesity were based on the new International Diabetes Federation definition

or claim codes for the individual outcome variables are provided in Online Resource 1.

\section{Statistical Analysis}

Continuous variables were presented as mean values with standard deviation and categorical variables as numbers with percentages. Continuous variables were compared using the Student $t$ test or the Wilcoxon rank-sum test, and categorical variables were compared using $\chi^{2}$ statistics or Fisher's exact test, as appropriate.

Incidence rates for the outcomes of interest were calculated as the number of first events divided by the total follow-up person-time at risk until the event or the end of 3-year follow-up, expressed as rates per 100 person-years. Cumulative incidence and survival curves were generated using the Kaplan-Meier method.

Multivariable Cox-proportional hazard regression models were applied to calculate hazard ratios (HRs) and 95\% confidence intervals (CIs) for the primary and secondary outcomes and for all-cause mortality, according to statin dose. The models included adjustment for age, gender, renal function (estimated glomerular filtration rate $[$ eGFR] $<60 \mathrm{~mL} /$ $\min / 1.73 \mathrm{~m}^{2}$ ), hypertension, diabetes, current smoking, and medications (including non-statin lipid-lowing agents, aspirin or P2Y12 inhibitors, beta-blockers, and RAAS inhibitors). Cox-proportional hazard regression models were also used to identify risk factors contributing to the primary and secondary outcomes and to all-cause mortality.

A two-tailed $p$ value $<0.05$ was considered statistically significant. Statistical analyses were performed using R software version 3.2.2 (R Foundation for Statistical Computing, Vienna, Austria; www.r-project.org).

\section{Results}

Among subjects enrolled in the AMC-HR between 1 January 2000 and 30 November 2013, a total of 32,850 had a history of clinically evident ASCVD and at least 1 major risk factor or at least 2 minor risk factors. After screening, 17,030 of these patients were excluded because they were aged $<40$ or $>85$ years, had a recent MI or stroke, did not have blood LDL-C levels available, were not receiving a statin, had uncontrolled hypertension, or had a history of hemorrhagic stroke. This resulted in a final study cohort of 15,820 patients, comprising 5419 with a history of MI, 8950 with a history of IS, and 1451 with SPAD (Fig. 1).

The baseline characteristics of the population are summarized in Table 2. In general, the characteristics of the AMC-HR cohort were consistent with those of the FOURIER population, with a few exceptions. A history of MI was less common in AMC-HR than in FOURIER $(34.3 \%$ versus $81.3 \%$ in the FOURIER placebo group), whereas a history of IS was more common (56.6\% versus $19.2 \%)$. 
Fig. 1 Flow of study participants. AMC, Asan Medical Center; IS, ischemic stroke; LDL-C, low-density lipoprotein cholesterol; MI, myocardial infarction; sPAD, symptomatic peripheral artery disease

\section{Electronic Medical Record}

AMC-HEART REGISTRY

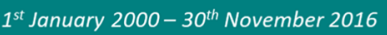
$\mathrm{N}=\mathbf{5 7 1 , 1 7 5}$

Screened with a history of clinically evident cardiovascular disease and at least 1 major risk factor or at least 2 minor risk factors between $1^{\text {st }}$ January 2000 and 30

\begin{tabular}{|c|c|c|c|c|}
\hline $\begin{array}{c}\text { Total } \\
\mathrm{N}=32,851\end{array}$ & $\begin{array}{c}M I \\
N=8,680\end{array}$ & $\begin{array}{c}\text { IS } \\
\mathrm{N}=21,242\end{array}$ & $\begin{array}{c}\text { SPAD } \\
N=2,928\end{array}$ & Excluded \\
\hline $1,893(5.8 \%)$ & $280(3.2 \%)$ & $1,447(6.8 \%)$ & $166(5.7 \%)$ & $\begin{array}{c}<40 \text { or }>85 \text { years of } \\
\text { age at time of } \\
\text { informed consent }\end{array}$ \\
\hline $766(2.3 \%)$ & $460(5.3 \%)$ & 304 (1.4\%) & $2(0.1 \%)$ & $\begin{array}{l}\text { Recent MI or } \\
\text { stroke within } 4 \\
\text { weeks }\end{array}$ \\
\hline $5,093(15.5 \%)$ & $941(10.8 \%)$ & 3,665 (17.3\%) & $487(16.6 \%)$ & LDL-C not available \\
\hline 8,853 (26.9\%) & $1,521(17.5 \%)$ & $6,532(30.8 \%)$ & $800(27.3 \%)$ & Statin not used \\
\hline $151(0.5 \%)$ & $23(0.3 \%)$ & $121(0.6 \%)$ & $7(0.2 \%)$ & $\begin{array}{l}\text { Uncontrolled } \\
\text { hypertension }\end{array}$ \\
\hline $274(0.8 \%)$ & $36(0.4 \%)$ & $223(1.0 \%)$ & $15(0.5 \%)$ & $\begin{array}{c}\text { Known } \\
\text { hemorrhagic } \\
\text { stroke at any time }\end{array}$ \\
\hline $\begin{array}{c}\text { Total } \\
\mathrm{N}=15,820\end{array}$ & $\begin{array}{c}\mathrm{Ml} \\
\mathrm{N}=5,419\end{array}$ & $\begin{array}{c}\text { IS } \\
N=8,950\end{array}$ & $\begin{array}{c}\text { SPAD } \\
N=1,451\end{array}$ & \\
\hline & $\begin{array}{r}\text { cal outcomes a } \\
\text { oint: all-cause mort } \\
\text { revascula }\end{array}$ & $\begin{array}{l}\text { years follow-up } \\
\mathrm{MI} \text {, stroke, UA or co } \\
\text { ion }\end{array}$ & & \\
\hline
\end{tabular}

Most patients in AMC-HR were receiving moderate-intensity statin therapy at the time of the index event (81.8\%), whereas in FOURIER most were on high-intensity statins (69.1\%). Mean LDL-C level was slightly higher in the AMC-HR cohort than in the FOURIER population (104 versus $92 \mathrm{mg} / \mathrm{dL}$ ), whereas triglyceride and lipoprotein(a) levels tended to be lower in the AMC-HR population. Mean body weight was lower in the AMC-HR cohort than in FOURIER $(64.9 \pm 14.6 \mathrm{~kg}$ versus $85.5 \pm 17.4 \mathrm{~kg}$ in the FOURIER placebo group).

The baseline characteristics for subcohorts of the AMCHR population, based on index cardiovascular event or on intensity of statin treatment are summarized in Online Resource 2. Patients in the subcohort with a history of MI were less likely to have hypertension than the subcohorts with a history of IS or SPAD $(68.3 \%$ versus $78.7 \%$ and $78.3 \%$ ) and more likely to be receiving beta-blockers
(90.5\% versus $49.7 \%$ and $68.3 \%$ ) and RAAS inhibitors (79.4\% versus $67.7 \%$ and $72.7 \%$ ). The IS subcohort had a numerically higher proportion of women $39.4 \%$ versus $24.0 \%$ and $22.5 \%$ ), a higher median LDL-C level (109 versus 99.4 and $95.4 \mathrm{mg} / \mathrm{dL}$ ), and a smaller proportion on ezetimibe (9.1\% versus $15.5 \%$ and $18.0 \%$ ) than the MI and SPAD subcohorts. The SPAD subcohort had a greater proportion of patients with diabetes $(55.8 \%$ versus $39.3 \%$ and $41.5 \%$ ) and patients receiving low-intensity statins (20.1\% versus $10.9 \%$ and $11.6 \%$ ) compared with the MI and IS subcohorts. The subcohorts who were receiving low-/moderate-intensity statin therapy had a higher median LDL-C level (105 versus $99.8 \mathrm{mg} / \mathrm{dL}$ ), lower triglyceride level (117 versus $123 \mathrm{mg} / \mathrm{dL}$ ), greater proportion of patients with a history of IS (59.1\% versus $45.1 \%)$, and smaller proportion on beta-blocker treatment $(63.3 \%$ 
Table 2 Baseline characteristics for the FOURIER-like AMC-HR study population and for the FOURIER study population

\begin{tabular}{|c|c|c|c|}
\hline & \multirow{2}{*}{$\begin{array}{l}\text { AMC-HR study (FOURIER-like } \\
\text { population) } \\
\text { Total }(n=15,820)\end{array}$} & \multicolumn{2}{|l|}{ FOURIER study [13] } \\
\hline & & Evolocumab $(n=13,784)$ & Placebo $(n=13,780)$ \\
\hline Age, years, mean $\pm S D$ & $63.2 \pm 10.2$ & $62.5 \pm 9.1$ & $62.5 \pm 8.9$ \\
\hline Men & $10,665(67.4)$ & $10,397(75.4)$ & $10,398(75.5)$ \\
\hline Weight, $\mathrm{kg}$, mean \pm SD & $64.9 \pm 14.6$ & $85.0 \pm 17.3$ & $85.5 \pm 17.4$ \\
\hline $\mathrm{BMI}, \mathrm{kg} / \mathrm{m}^{2}$, mean $\pm \mathrm{SD}$ & $24.7 \pm 8.4$ & - & - \\
\hline $\mathrm{SBP}, \mathrm{mmHg}$, mean $\pm \mathrm{SD}$ & $129.7 \pm 21.5$ & - & - \\
\hline $\mathrm{DBP}, \mathrm{mmHg}$, mean $\pm \mathrm{SD}$ & $74.8 \pm 12.5$ & & \\
\hline $\mathrm{eGFR}<60 \mathrm{~mL} / \mathrm{min} / 1.73 \mathrm{~m}^{2}$ & $2773(17.6)$ & - & - \\
\hline \multicolumn{4}{|l|}{ Lipids, median (IQR) } \\
\hline $\mathrm{LDL}-\mathrm{C}, \mathrm{mg} / \mathrm{dL}$ & $104(78-132)$ & $92(80-109)$ & $92(80-109)$ \\
\hline Total cholesterol, mg/dL & $164(134-197)$ & $168(151-188)$ & $168(151-189)$ \\
\hline HDL-C, mg/dL & $42(35-51)$ & $44(37-53)$ & $44(37-53)$ \\
\hline Triglycerides, mg/dL & $118(85-166)$ & $134(101-183)$ & $133(99-181)$ \\
\hline Lipoprotein(a), nmol/L & $23.3(12.1-42.5)$ & $37(13-166)$ & $37(13-164)$ \\
\hline \multicolumn{4}{|l|}{ Cardiovascular risk factors } \\
\hline Hypertension & $11,881(75.1)$ & $11,045(80.1)$ & $11,039(80.1)$ \\
\hline Diabetes & $6651(42.0)$ & $5054(36.7)$ & $5027(36.5)$ \\
\hline Current smoking & $3439(21.7)$ & $3854(28.0)$ & $3923(28.5)$ \\
\hline \multicolumn{4}{|l|}{ Cardiovascular disease } \\
\hline MI & $5419(34.3)$ & $11,145(80.9)$ & $11,206(81.3)$ \\
\hline IS & $8950(56.6)$ & $2686(19.5)$ & 2651(19.2) \\
\hline sPAD & $1451(9.2)$ & $1858(13.5)$ & $1784(12.9)$ \\
\hline \multicolumn{4}{|l|}{ Statin use: statin intensity at the index event } \\
\hline Low intensity & $1919(12.1)$ & $38(0.3)$ & $31(0.2)$ \\
\hline Moderate intensity & $12,936(81.8)$ & $4161(30.2)$ & $4231(30.7)$ \\
\hline High intensity & $965(6.1)$ & $9585(69.5)$ & $9518(69.1)$ \\
\hline \multicolumn{4}{|l|}{ Other medication use } \\
\hline \multicolumn{4}{|l|}{ Non-statin lipid-lowering treatment } \\
\hline Ezetimibe & $1919(12.1)$ & $726(5.3)$ & $714(5.2)$ \\
\hline Fibrate & $927(5.9)$ & - & - \\
\hline Niacin & $0(0.0)$ & - & - \\
\hline Cholestyramine & $17(0.1)$ & - & - \\
\hline Aspirin or P2Y12 inhibitors & $14,904(94.2)$ & $12,766(92.7)$ & $12,666(92.0)$ \\
\hline Beta-blockers & $10,340(65.4)$ & $10,441(75.8)$ & $10,374(75.4)$ \\
\hline $\begin{array}{l}\text { RAAS inhibitors (ACE inhibitors or ARBs, } \\
\text { aldosterone antagonists, or both) }\end{array}$ & $11,420(72.2)$ & $10,803(78.4)$ & $10,730(77.9)$ \\
\hline
\end{tabular}

Data are presented as $n(\%)$ unless otherwise indicated

$A C E$ angiotensin-converting enzyme, $A C R$ albumin-to-creatinine ratio, $A M C$ Asan Medical Center Heart Registry, $A R B$ angiotensin-receptor blocker, $B M I$ body mass index, $B P$ blood pressure, $C A B G$ coronary artery bypass graft, $e G F R$ estimated glomerular filtration rate, $H D L-C$ highdensity lipoprotein cholesterol, $h S C R P$ high-sensitivity C-reactive protein, $I Q R$ interquartile range, $I S$ ischemic stroke, $L D L-C$ low-density lipoprotein cholesterol, $M I$ myocardial infarction, $P C I$ percutaneous coronary intervention, $R A A S$ renin-angiotensin-aldosterone system, $S B P$ systolic blood pressure, $S D$ standard deviation, $S P A D$ symptomatic peripheral artery disease

versus $74.7 \%$ ), compared with the subcohorts who were receiving high-intensity statins or statins plus ezetimibe.

\section{Cardiovascular Events}

The cumulative incidences of the primary and secondary endpoints are summarized in Fig. 2, for the overall AMC-HR cohort and for the subcohorts with a history of MI, IS, or sPAD. For the total cohort, the 3-year cumulative incidence of the composite primary endpoint (MI, stroke, hospitalization for UA, coronary revascularization, and all-cause mortality) was $15.3 \%$, and the 3 -year cumulative incidence of the secondary composite endpoint (MI, stroke, and all-cause mortality) was $6.0 \%$. The 3 -year cumulative incidences of 


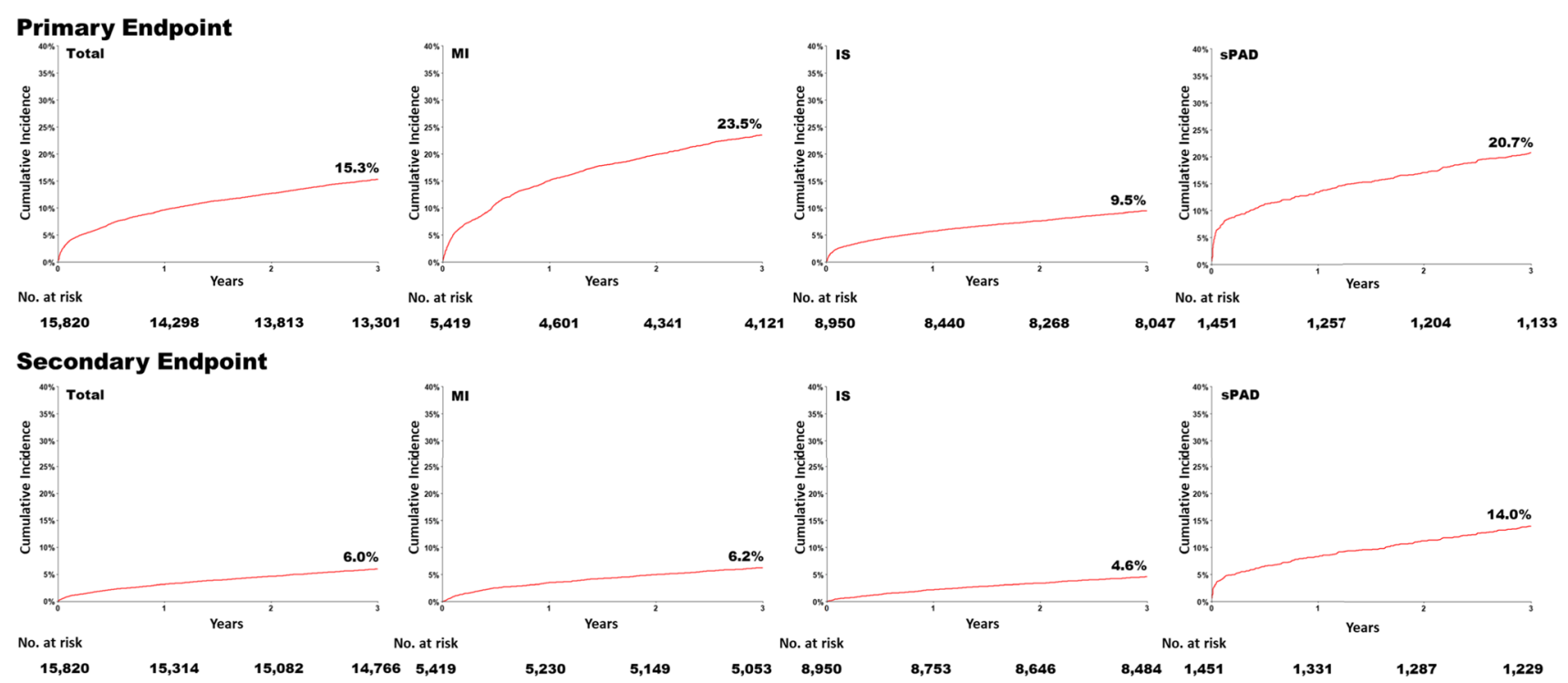

Fig. 2 Three-year cumulative incidences of the primary and secondary endpoints in AMC-HR (for the total cohort and for the subcohorts with a history of MI, IS, or SPAD). Primary endpoint: composite of MI, stroke, hospitalization for UA, revascularization, and all-cause mortality. Secondary endpoint: composite of MI, stroke, and all-cause mortality. IS, ischemic stroke; MI, myocardial infarction; sPAD, symptomatic peripheral artery disease; UA, unstable angina the composite primary and secondary endpoints were $23.5 \%$ and $6.2 \%$ in the MI subcohort, $9.5 \%$ and $4.6 \%$ in the IS subcohort, and $20.7 \%$ and $14.0 \%$ in the SPAD subcohort.

Cardiovascular event rates are presented in Table 3. For the overall AMC-HR cohort, the 3-year incidence rate for the primary composite endpoint was 5.7 (95\% CI 5.5-5.9) per 100 person-years and for the secondary composite endpoint was 2.1 (95\% CI 2.0-2.2) per 100 person-years. Among the individual components of the primary endpoint, 3-year incidence rates in the overall cohort ranged from 0.4 (95\% CI 0.3-0.4) for MI to 2.8 (95\% CI 2.6-2.9) for revascularization procedures. After stratification by subcohort, revascularization and hospitalization for UA was most frequent in the MI cohort, while stroke and all-cause mortality were the second most frequent events in the SPAD and IS cohorts, respectively.

The risk of meeting the primary or secondary endpoints during the 3 years of observation did not differ between recipients of different intensities of statin therapy (Table 4). Nonetheless, there was a trend towards a reduced risk of all-cause mortality in high-intensity treatment, as indicated by hazard ratios of 0.66 (95\% CI 0.38-1.13) for high- versus low-intensity statin use and 0.73 (95\% CI $0.50-1.07)$ for high-intensity statin or any statin plus ezetimibe versus low-/ moderate-intensity statin without ezetimibe. No association between the baseline LDL-C level and recurrent cardiovascular events was observed (Table 4).

\section{Risk Factors}

Results of the analysis of potential risk factors contributing to cardiovascular outcomes within 3 years in the AMCHR cohort are summarized in Table 5. Factors significantly associated with an increased risk of meeting the primary endpoint included male sex, diabetes, reduced renal function (eGFR $<60 \mathrm{~mL} / \mathrm{min} / 1.73 \mathrm{~m}^{2}$ ), and receiving non-statin lipid-lowering treatment, aspirin or P2Y12 inhibitors, betablockers, or RAAS inhibitors. Factors associated with an increased risk of meeting the secondary endpoint included age $\geq 65$ years, diabetes, eGFR $<60 \mathrm{~mL} / \mathrm{min} / 1.73 \mathrm{~m}^{2}$, and treatment with beta-blockers or RAAS inhibitors. Factors significantly associated with an increased risk of all-cause mortality included age $\geq 65$ years, diabetes, eGFR $<60 \mathrm{~mL} /$ $\min / 1.73 \mathrm{~m}^{2}$, and treatment with beta-blockers.

\section{LDL-C Follow-Up}

LDL-C levels were re-evaluated within 1 year of the index event in $60.8 \%$ of the AMC-HR cohort, with no marked difference seen between subgroups based on the intensity of statin treatment that was being received at the time of the index event (Table 6). LDL-C goals were only achieved in $24.4 \%$ of patients within a year; there was a trend towards a higher rate of goal achievement as statin intensity increased (from $18.7 \%$ in the low-intensity group to $34.1 \%$ in the highintensity group). Lipid-lowering treatment was intensified in only $0.7 \%$ of patients during 1 year of follow-up. 


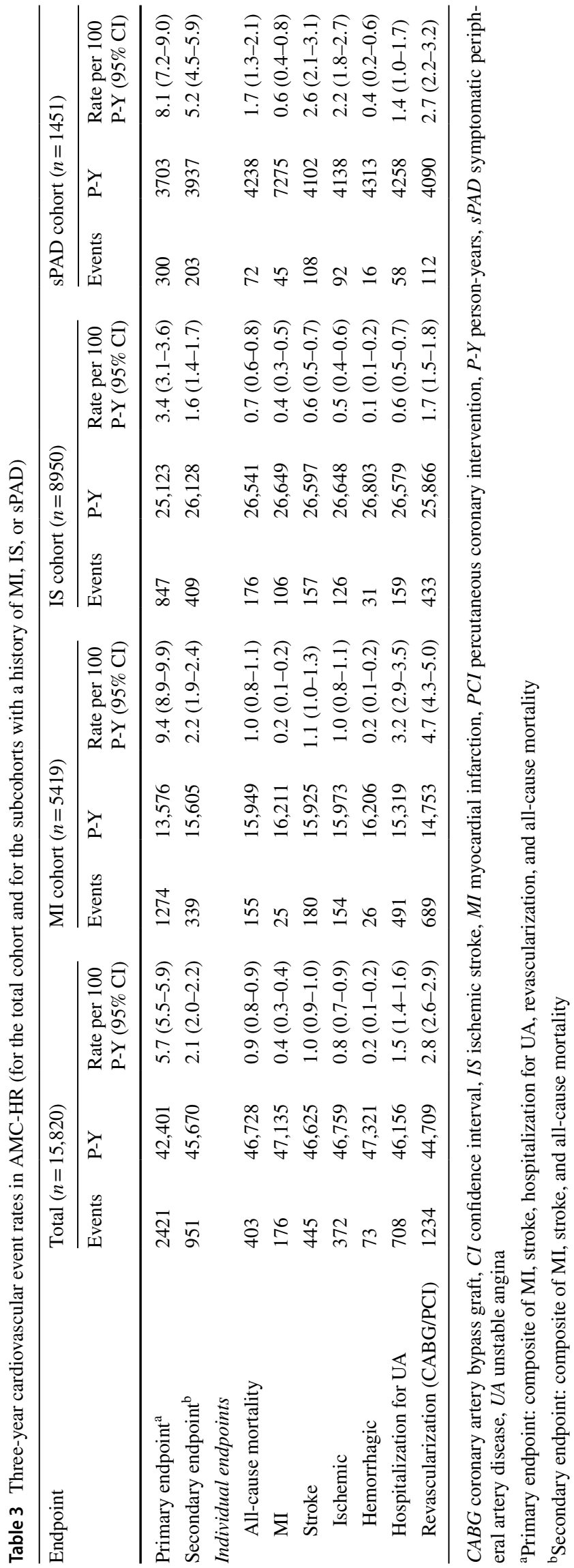

\section{Discussion}

In the current study using EMR data, we aimed to estimate the risk of cardiovascular outcomes among very high-risk Korean patients. The main finding was that the risk of further recurrent cardiovascular events was considerable in very high-risk Korean ASCVD patients managed in a routine practice setting as a 3-year incidence rate for the primary composite endpoint of 5.7 per 100 person-years (cumulative incidence of $15.3 \%$ ). No significant relationship was found between statin intensity and the risk of meeting the study's primary or secondary endpoints, where the majority used a moderate-intensity statin. In addition, suboptimal LDL-C management during the follow-up period was observed even on statin treatment.

The 3-year cumulative incidence of $15.3 \%$ for this endpoint was consistent with that seen in the placebo group of the FOURIER clinical trial (14.6\%) [13], although the primary endpoints in these studies differed slightly, in that the AMCHR study included all-cause mortality, whereas FOURIER included only cardiovascular death. A few other retrospective studies have also assessed cardiovascular events in a FOURIER-like population in a real-world setting. In a US study, the cumulative incidence of the primary composite endpoint (MI, stroke, coronary revascularization, and cardiovascular death [but not hospitalization for UA]) after 2.2 years was $12.2 \%$, and the cardiovascular event rate was approximately 6.0 per 100 person-years [14]. A Swedish study found that $43.8 \%$ of patients experienced one or more new events (using the same composite primary endpoint as FOURIER) during a mean follow-up of 7.3 years, with an incidence rate of 7.0 per 100 person-years [15]. A study in the UK found that 33\% of patients experienced a new cardiovascular event (same composite primary endpoint as FOURIER) during a mean follow-up of 5.4 years, with an incidence rate of 7.5 per 100 person-years [16]. With previous literatures, the present study adds to the body of evidence from a real-world setting on the high risk of further cardiovascular events in patients with clinically evident ASCVD and the requirement for additional therapeutic options.

There were a few noteworthy differences between the present cohort driven from AMC-HR and the FOURIER trial population. Firstly, there was a higher prevalence of IS and a lower prevalence of MI in the present cohort compared with FOURIER. It is well-known that Asians have a higher prevalence/incidence of IS than MI $[17,18]$. Of note, it can be assumed that if the prevalence of MI in the present cohort had been similar to that in FOURIER, the rate of recurrent events of all population would have been higher than was actually observed because recurrent events occurred more frequently in the MI subgroup compared with other subgroups in AMC-HR cohorts. Secondly, most 


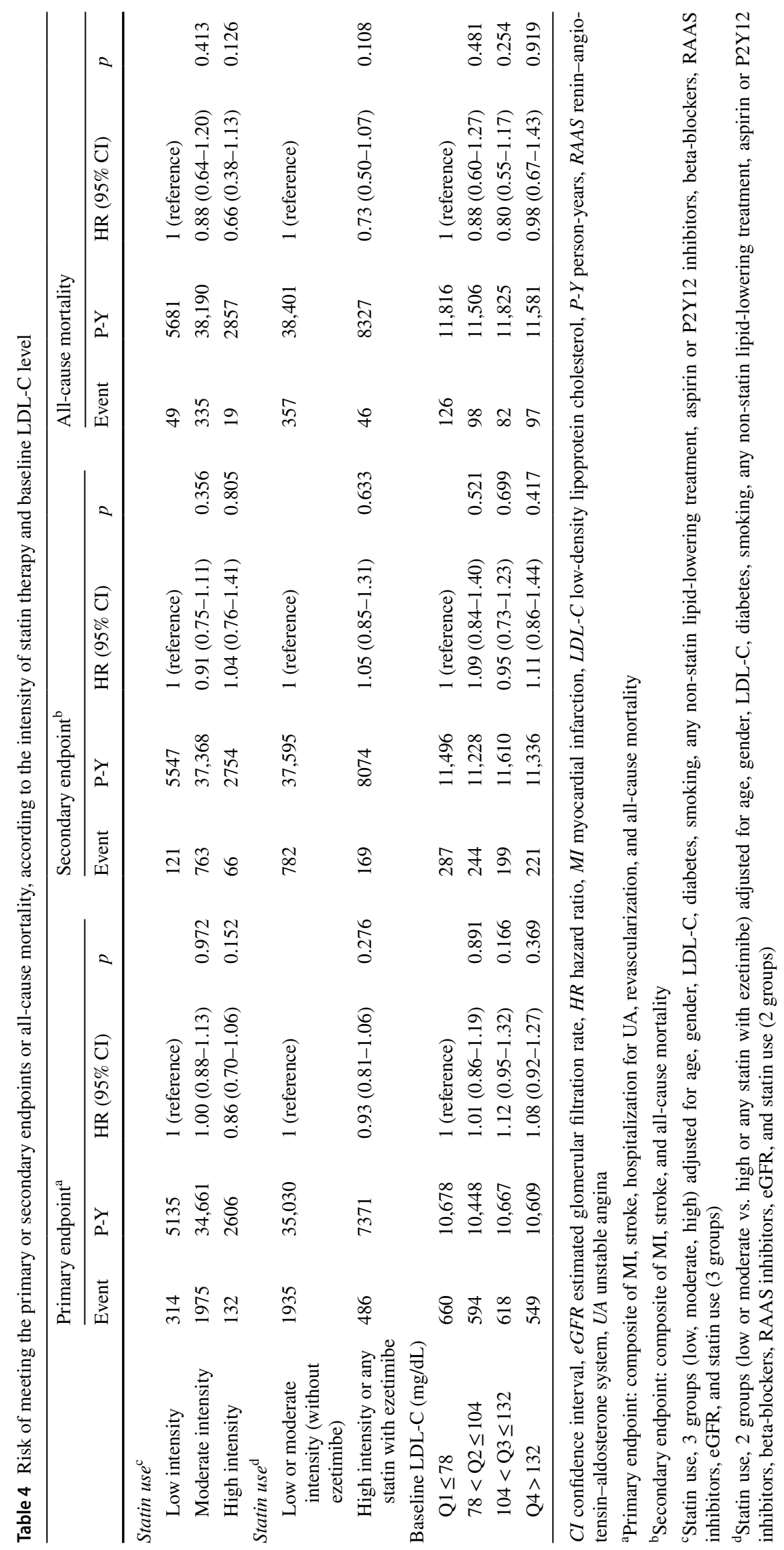




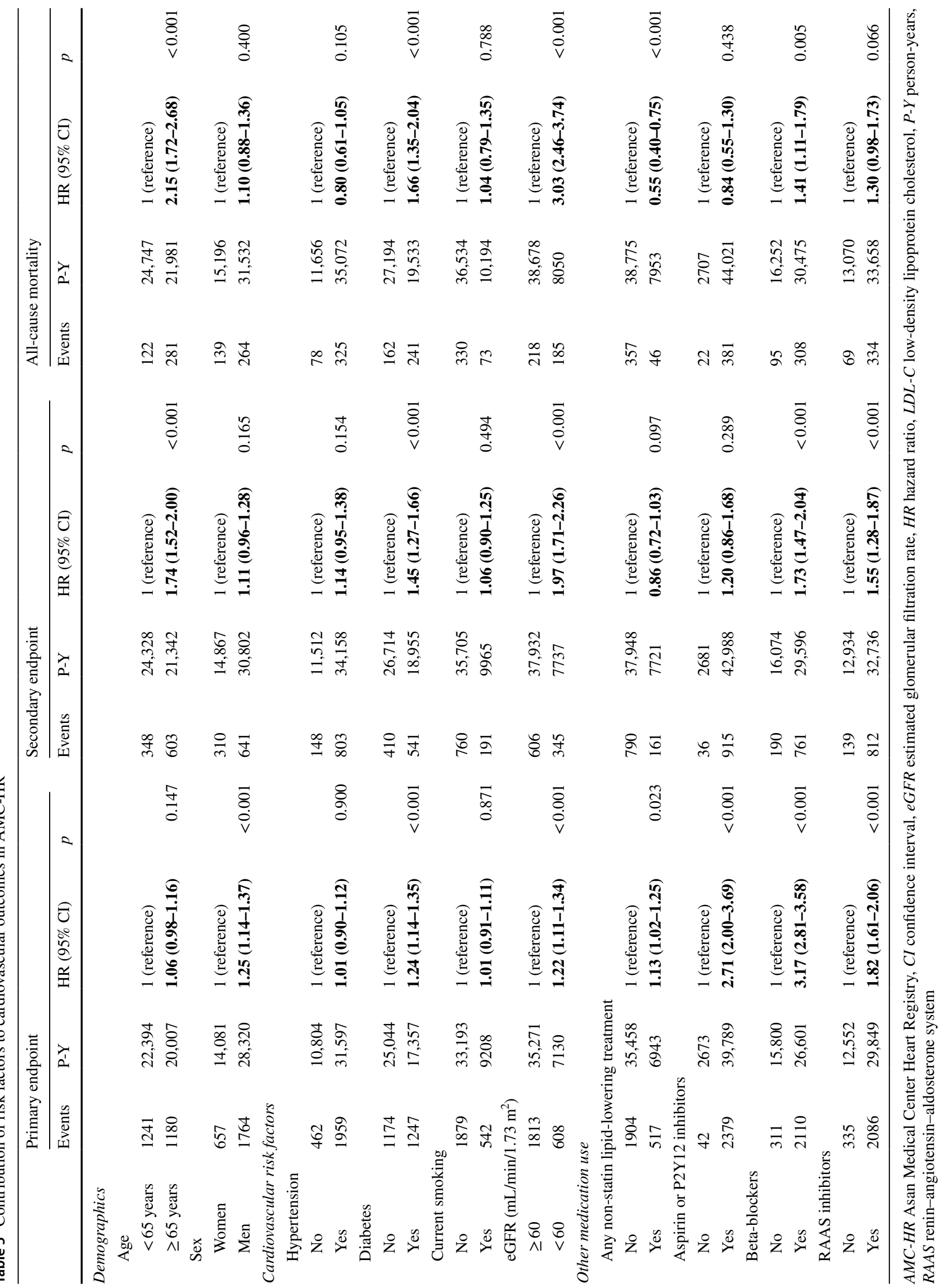


Table 6 LDL-C follow-up and alterations in lipid-lowering treatment, according to statin intensity at time of the index event

\begin{tabular}{|c|c|c|c|c|}
\hline & $\begin{array}{l}\text { Total population } \\
n=15,820\end{array}$ & $\begin{array}{l}\text { Low-intensity } \\
n=1919\end{array}$ & $\begin{array}{l}\text { Moderate-intensity } \\
n=12,936\end{array}$ & $\begin{array}{l}\text { High-intensity } \\
n=965\end{array}$ \\
\hline LDL-C follow-up between 2 weeks and 1 year & $9613(60.8 \%)$ & $1124(58.6 \%)$ & $7911(61.2 \%)$ & $578(59.9 \%)$ \\
\hline $\mathrm{LDL}-\mathrm{C}<70 \mathrm{mg} / \mathrm{dL}$ or $50 \%$ reduction & $3867(24.4 \%)$ & $359(18.7 \%)$ & $3179(24.6 \%)$ & $329(34.1 \%)$ \\
\hline Statin dose increased or ezetimibe added & $103(0.7 \%)$ & $29(1.5 \%)$ & $73(0.6 \%)$ & $1(0.1 \%)$ \\
\hline
\end{tabular}

Data are presented as $n(\%)$

$L D L-C$ low-density lipoprotein cholesterol

patients $(81.8 \%)$ in the present study were receiving moderate-intensity statin therapy at the time of the index event, whereas in FOURIER, approximately $70 \%$ of patients were receiving high-intensity statin treatment. In fact, the high prevalence of moderate-intensity statin treatment resulting in suboptimal LDL cholesterol reduction might importantly contribute to the lack of association between statin intensity and clinical outcomes in AMC-HR patients. However, interpretation should be cautious since the majority of patients received moderate statin, leading to statistical limitations for pair comparison. On the other hand, East Asian patients achieve a response to statins at a lower dose than required for Caucasians. Consequently, moderate-intensity statin therapy may be sufficient in most East Asian patients, with high-intensity statin therapy required less often $[19,20]$. Hence, no significant relationship between statin intensity and the risk of endpoints in the present study may partly be explained by the ethnic disparity.

Management of LDL-C levels is important for the prevention of ASCVD; however, underutilization of lipidlowering treatment and failure to intensify treatment is common, even for patients at high cardiovascular risk [21-26]. Measurement of LDL-C levels is often not performed in patients hospitalized with IS, even among those at high risk of future events [25, 27, 28]. Few data are available about the frequency with which lipid levels are measured during follow-up of very high-risk patients receiving lipid-lowering treatment in real-world practice. In the present study based upon EMR data, during the initial screen process, $26.9 \%$ of patients were excluded because they were non-statin users (Fig. 1). In addition, during the follow-up period, LDL-C levels were re-evaluated within 1 year in only $60.8 \%$ of patients and only $24.4 \%$ of patients achieved LDL-C goals within that time. Despite this, $<1 \%$ of patients received intensification of statin therapy. These findings suggest inadequate followup of LDL-C levels and highlight the need to provide better lipid monitoring. In addition, the substantially low achievement rate of LDL reduction, similarly reported in the previous investigations, indicates that new therapies such as PCSK9 inhibitors may provide an opportunity to reduce the burden of cardiovascular events among the very high ASCVD population [29, 30].
Several limitations should be noted. The first of these was inherent to the retrospective nature and observational design of the analyses. Confirmation of the results in a prospective study would be helpful. Second, although EMR data enabled reliable real-world estimates, findings from a single center cannot be generalized. Third, on analysis of risk factors for primary and secondary endpoints, cardiovascular protective medications such as aspirin, P2Y12 inhibitors, betablockers, or RAAS inhibitors were associated with recurrent events. These results may be due to inherent biases of retrospective analysis, but similar findings were also observed in other retrospective studies [14]. It is reasonable to assume that higher risk patients with comorbidities prone to further cardiovascular events were treated more aggressively with various kinds of cardioprotective medications. Additional methodologically rigorous studies are needed to investigate the risk factors on cardiovascular outcomes in a real-world setting. Finally, the event rate was potentially underestimated because clinical events were captured from a singlecenter EMR database. Linking it with national claims data or health insurance data might possibly capture the events more accurately.

\section{Conclusion}

This study in a routine practice setting in Korea found that very high-risk patients with clinically evident ASCVD were at substantial risk of further cardiovascular events within the subsequent 3 years. In addition, suboptimal LDL-C reduction, even on statin treatment, was observed. These findings may highlight the unmet need and clinical burden among very high-risk ASCVD patients treated with statin therapy and indicate the requirement for additional therapeutic options such as PCSK9 inhibitors.

Supplementary Information The online version contains supplementary material available at https://doi.org/10.1007/s10557-021-07255-2.

Acknowledgements Under the direction of the authors, Kathy Croom and David P. Figgitt, PhD, ISMPP CMPPTM, Content Ed Net, provided medical writing and editorial assistance in the preparation of the manuscript, with funding from Amgen Korea. 
Author Contribution Osung Kwon conceived and designed the analysis, performed the analysis, and wrote the paper; Wonjun Na collected data, contributed data or analysis tools, and wrote the paper; Jaehee Hur collected data and contributed data or analysis tools; Ju Hyeon Kim contributed data or analysis tools and performed the analysis; Tae Joon Jun contributed data or analysis tools and performed the analysis; Heejun Kang contributed data or analysis tools and performed the analysis and statistical validation; Hojoon Lee performed the analysis and wrote the paper; Young-Hak Kim conceived and designed the analysis and performed the analysis.

Funding This study was funded by Amgen Korea.

Availability of Data and Material Not applicable.

\section{Declarations}

Ethics Approval/Research Involving Human Participants For this retrospective observational study, collection of de-identified data from the AMC Heart Registry based on implied consent was approved by the Asan Medical Center institutional review board. De-identification of data was performed in accordance with the health insurance portability and accountability act in Korea.

Consent to Participate Implied consent was provided due to participation in the AMC Heart Registry.

\section{Consent for Publication Not applicable.}

Conflict of Interest Hojoon Lee reports employment by Amgen Korea. All other authors have nothing to disclose.

Open Access This article is licensed under a Creative Commons Attribution 4.0 International License, which permits use, sharing, adaptation, distribution and reproduction in any medium or format, as long as you give appropriate credit to the original author(s) and the source, provide a link to the Creative Commons licence, and indicate if changes were made. The images or other third party material in this article are included in the article's Creative Commons licence, unless indicated otherwise in a credit line to the material. If material is not included in the article's Creative Commons licence and your intended use is not permitted by statutory regulation or exceeds the permitted use, you will need to obtain permission directly from the copyright holder. To view a copy of this licence, visit http://creativecommons.org/licenses/by/4.0/.

\section{References}

1. GBD 2016 Causes of Death Collaborators. Global, regional, and national age-sex specific mortality for 264 causes of death, 1980-2016: a systematic analysis for the Global Burden of Disease Study 2016. Lancet. 2017;390(10100):1151-210.

2. Li S, Peng Y, Wang X, et al. Cardiovascular events and death after myocardial infarction or ischemic stroke in an older Medicare population. Clin Cardiol. 2019;42(3):391-9.

3. Smolina K, Wright FL, Rayner M, Goldacre MJ. Long-term survival and recurrence after acute myocardial infarction in England, 2004 to 2010. Circ Cardiovasc Qual Outcomes. 2012;5(4):532-40.

4. Hillen T, Coshall C, Tilling K, Rudd AG, McGovern R, Wolfe $\mathrm{CD}$, South London Stroke Register. Cause of stroke recurrence is multifactorial: patterns, risk factors, and outcomes of stroke recurrence in the South London Stroke Register. Stroke. 2003;34(6):1457-63.

5. Punekar RS, Fox KM, Richhariya A, et al. Burden of first and recurrent cardiovascular events among patients with hyperlipidemia. Clin Cardiol. 2015;38(8):483-91.

6. Wong ND. Epidemiological studies of CHD and the evolution of preventive cardiology. Nat Rev Cardiol. 2014;11(5):276-89.

7. Nelson RH. Hyperlipidemia as a risk factor for cardiovascular disease. Prim Care. 2013;40(1):195-211.

8. Jellinger PS, Handelsman Y, Rosenblit PD, et al. American Association of Clinical Endocrinologists and American College of Endocrinology Guidelines for Management of Dyslipidemia and Prevention of Cardiovascular Disease. Endocr Pract. 2017;23(Suppl 2):1-87.

9. National Cholesterol Education Program (NCEP) Expert Panel on Detection, Evaluation, and Treatment of High Blood Cholesterol in Adults (Adult Treatment Panel III). Third Report of the National Cholesterol Education Program (NCEP) Expert Panel on Detection, Evaluation, and Treatment of High Blood Cholesterol in Adults (Adult Treatment Panel III) final report. Circulation. 2002;106(25):3143-421.

10 Ference BA, Ginsberg HN, Graham I, et al. Low-density lipoproteins cause atherosclerotic cardiovascular disease. 1. Evidence from genetic, epidemiologic, and clinical studies. A consensus statement from the European Atherosclerosis Society Consensus Panel. Eur Heart J. 2017;38(32):2459-72.

11. Collins R, Reith C, Emberson J, et al. Interpretation of the evidence for the efficacy and safety of statin therapy. Lancet. 2016;388(10059):2532-61.

12. Koskinas KC, Siontis GCM, Piccolo R, et al. Effect of statins and non-statin LDL-lowering medications on cardiovascular outcomes in secondary prevention: a meta-analysis of randomized trials. Eur Heart J. 2018;39(14):1172-80.

13. Sabatine MS, Giugliano RP, Keech AC, FOURIER Steering Committee and Investigators, et al. Evolocumab and clinical outcomes in patients with cardiovascular disease. N Engl J Med. 2017;376(18):1713-22.

14. Colantonio LD, Monda KL, Rosenson RS, et al. Characteristics and cardiovascular disease event rates among African Americans and whites who meet the Further cardiovascular OUtcomes Research with PCSK9 Inhibition in subjects with Elevated Risk (FOURIER) trial inclusion criteria. Cardiovasc Drugs Ther. 2019;33(2):189-99.

15. Lindh M, Banefelt J, Fox KM, et al. Cardiovascular event rates in a high atherosclerotic cardiovascular disease risk population: estimates from Swedish population-based register data. Eur Heart J Qual Care Clin Outcomes. 2019;5(3):225-32.

16. Toth PP, Danese M, Villa G, et al. Estimated burden of cardiovascular disease and value-based price range for evolocumab in a high-risk, secondary-prevention population in the US payer context. J Med Econ. 2017;20(6):555-64.

17. Shin SY, Kim WS, Lee JH. Characteristics desired in clinical data warehouse for biomedical research. Healthc Inform Res. 2014;20(2):109-16.

18. Ahn I, Na W, Kwon O, et al. CardioNet: a manually curated database for artificial intelligence-based research on cardiovascular diseases. BMC Med Inform Decis Mak. 2021;21(1):29.

19. Liao JK. Safety and efficacy of statins in Asians. Am J Cardiol. 2007;99(3):410-4.

20. Kim HS, Lee H, Lee SH, et al. Use of moderate-intensity statins for low-density lipoprotein cholesterol level above 190 $\mathrm{mg} / \mathrm{dL}$ at baseline in Koreans. Basic Clin Pharmacol Toxicol. 2017;121(4):272-8.

21. Ferrieres J, De Ferrari GM, Hermans MP, et al. Predictors of LDL-cholesterol target value attainment differ in acute and 
chronic coronary heart disease patients: results from DYSIS II Europe. Eur J Prev Cardiol. 2018;25(18):1966-76.

22. Tan NC, Goh CC, Goh SC, Koh YL, Koh KH. The effect of the intensity of lipid-lowering medications on the LDL cholesterol treatment goals of Asian patients with dyslipidaemia in primary care. J Clin Pharm Ther. 2016;41(6):677-83.

23. Nanna MG, Navar AM, Wang TY, et al. Practice-level variation in statin use and low-density lipoprotein cholesterol control in the United States: results from the Patient and Provider Assessment of Lipid Management (PALM) registry. Am Heart J. 2019;214:113-24.

24. Chiang CE, Ferrières J, Gotcheva NN, et al. Suboptimal control of lipid levels: results from 29 countries participating in the centralized pan-regional surveys on the undertreatment of hypercholesterolaemia (CEPHEUS). J Atheroscler Thromb. 2016;23(5):567-87.

25. Ovbiagele B, Hills NK, Saver JL, Johnston SC, CASPR Investigators. Lipid assessment and treatment patterns in hospitalized TIA and ischemic stroke patients. J Hosp Med. 2006;1(4):214-20.

26. Saposnik G, Fonarow GC, Pan W, AHA Get-with-the-Guidelines Stroke, et al. Guideline-directed low-density lipoprotein management in high-risk patients with ischemic stroke: findings from Get with the Guidelines-Stroke 2003 to 2012. Stroke. 2014;45(11):3343-51.
27. Mullard AJ, Reeves MJ, Jacobs BS, Paul Coverdell National Acute Stroke Registry Michigan Prototype Investigators, et al. Lipid testing and lipid-lowering therapy in hospitalized ischemic stroke and transient ischemic attack patients: results from a statewide stroke registry. Stroke. 2006;37(1):44-9.

28. Ní Chróinín D, Ní Chróinín C, Akijian L, et al. Suboptimal lipid management before and after ischaemic stroke and TIA-the North Dublin Population Stroke Study. Ir J Med Sci. 2018;187(3):739-46.

29. Gitt AK, Lautsch D, Ferrières J, et al. Cholesterol target value attainment and lipid-lowering therapy in patients with stable or acute coronary heart disease: results from the Dyslipidemia International Study II. Atherosclerosis. 2017;266:158-66.

30. Yang YS, Yang BR, Kim MS, et al. Low-density lipoprotein cholesterol goal attainment rates in high-risk patients with cardiovascular diseases and diabetes mellitus in Korea: a retrospective cohort study. Lipids Health Dis. 2020;19(5):13.

Publisher's Note Springer Nature remains neutral with regard to jurisdictional claims in published maps and institutional affiliations. 